\title{
Non-thermal processes associated with rising structures and waves during a "halo" type CME`
}

\author{
N. J. Lehtinen ${ }^{1}$, S. Pohjolainen ${ }^{1}$, M. Karlický ${ }^{2}$, H. Aurass ${ }^{3}$, and W. Otruba ${ }^{4}$ \\ 1 Tuorla Observatory/VISPA, University of Turku, Väisäläntie 20, 21500 Piikkiö, Finland \\ e-mail: nijole@utu.fi \\ 2 Astronomical Institute of the Academy Sciences of the Czech Republic, 25165 Ondřejov, Czech Republic \\ 3 Astrophysical Institute Potsdam, 14482 Potsdam, Germany \\ ${ }^{4}$ Sonnenobservatorium Kanzelhöhe, 9521 Treffen, Austria
}

Received 31 May 2004 / Accepted 21 March 2005

\section{ABSTRACT}

We analyse structures and events connected with a flare-associated "halo" type coronal mass ejection (CME) observed on December 18, 2000. A GOES C7.0 class X-ray flare started at 11:02 UT in NOAA Active Region 9269, located at N14 E03. Yohkoh SXT observed slowly rising soft $\mathrm{X}$-ray loops already some $5 \mathrm{~min}$ before flare start. $\mathrm{H} \alpha$ images show a two-ribbon flare, remote brightenings, and a partly disappearing filament near the active region. A metric radio precursor was observed to start at 11:06:30 UT, simultaneously with impulsive emission in hard X-rays and microwaves. The frequency-drifting precursor envelope was superposed with J- and reverse drift bursts. The radio bursts traced large-scale soft X-ray loop structures about $160000 \mathrm{~km}$ away from the flare core, and hard X-ray emission was observed at the ends of some of these loops. The precursor emission points to a rising structure where electron acceleration takes place. Later on, a radio type II burst (signature of a propagating shock, driven either by an ejecta or a blast wave) and an EIT wave were observed. We conclude that possible sources for the rising structure and accelerator of electron beams are (1) large-scale loops that connect the flare core region and the precursor site in the close vicinity of two separate rising filaments, and (2) a growing shock that accelerates electrons along closed field lines until the multipolar field is opened and the CME is lifted off. As neither X-ray nor EUV ejecta could be observed whether in the direction of the type II burst or near the radio precursor, we find some support for the shock wave scenario.

Key words. Sun: corona - Sun: coronal mass ejections (CMEs) - Sun: filaments - Sun: flares - Sun: radio radiation Sun: X-rays, gamma rays

\section{Introduction}

"Halo" coronal mass ejections (CMEs) are gigantic plasmamagnetic field configurations that sometimes surround the screened solar disk in white-light coronagraph images. Halotype events are produced by CMEs that are directed towards the Earth (in case they are not occuring at the back side of the Sun), and have been found to be connected with geomagnetic disturbances and changes in the solar wind flow (see, e.g., Gosling 1997; Reames 1999; Gopalswamy et al. 2002). CMEs are associated with solar flares and prominence eruptions, but they can also occur in the absence of either one of these processes, and vice versa. If the halo CME-associated structures and events occur on the visible solar disk, they can be studied in detail. Recent studies have linked halo CMEs with EIT waves (Cliver et al. 1999; Thompson et al. 1999;

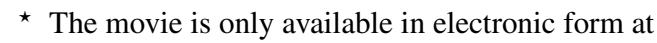
http://www. edpsciences.org
Biesecker et al. 2002), soft X-ray and EUV dimmings (Sterling $\&$ Hudson 1997; Zarro et al. 1999), H $\alpha$ (Moreton) waves (Pohjolainen et al. 2001), type II and type IV radio emission (Claßen \& Aurass 2002; Caroubalos et al. 2004), disappearing transequatorial loops (Khan \& Hudson 2000; Pohjolainen et al. 2001; Glover et al. 2003), and oscillating filaments (Moreton 1964; Eto et al. 2002; Ji et al. 2002).

"EIT waves" are probably large amplitude MHD waves (simple MHD waves, Mann 1995) in the corona, first observed with EIT on-board SOHO (Thompson et al. 1999). They are very closely associated with type II radio bursts, although the derived speeds have been found to be different (Klassen et al. 2000). A typical EIT wave is comparatively slow (200-300 $\mathrm{km} \mathrm{s}^{-1}$ ). This discrepancy is intrinsic in observation procedures and the physics of large amplitude waves, and has been explained by Warmuth et al. (2004a,b) - both observed wave phenomena can be understood as due to a common driving agent. It should be mentioned that EIT waves are also 
interpreted as a lower skirt of the CME sweeping through the corona (Delannée \& Aulanier 1999), a model which can be applied well in some cases.

Solar radio type II bursts are thought to be caused by MHD shock waves propagating through the corona and interplanetary medium (e.g. Nelson \& Melrose 1985). Both flare (blast) waves and piston-driven bow shocks could be the drivers. Only a small number of flares are accompanied by type II bursts, but almost all type II bursts are associated with flares and CMEs (see, e.g., Cliver et al. 1999). The frequency drifts of type II bursts are in the range of $0.1-1.0 \mathrm{MHz} \mathrm{s}^{-1}$, and speeds - with some uncertainty because of the density model for transforming drifts into speeds - are in the range of 400-2000 $\mathrm{km} \mathrm{s}^{-1}$ and on the average $700 \mathrm{~km} \mathrm{~s}^{-1}$ (Nelson $\&$ Melrose 1985). Type II bursts can be created only when the coronal conditions are favorable, i.e. when the Alfvén speed is especially low and $V_{r} \cdot \cos \alpha=1.4 v_{\mathrm{A}}$ is fulfilled (Mann et al. 2003). Radial velocity $V_{r}$ is related to the full velocity $V$ by $V_{r}=V / \cos \alpha$, where $\alpha$ is the angle between the straight line and radial direction (see Fig. 3 in Mann et al. 2003), and $v_{\mathrm{A}}$ is the Alfvén speed.

Some type II bursts have precursors, and their signatures occur during the impulsive flare phase and have been found to be located between the flare site and the type II position (Klassen et al. 2003). A precursor is defined as a sequence of fast-drift bursts where the frequency envelope drifts at a normalized drift rate, almost similar to the subsequent type II burst (Klassen et al. 1999).

It is well known that during type II bursts, at higher frequencies, a meter-decimeter continuum with spectral fine structures (pulsations, zebra pattern, fibers) can occur (Robinson 1985). In some eruptive flares, at frequencies higher than $800 \mathrm{MHz}$, drifting pulsating structures (DPS) are observed before and/or during type II radio bursts (Karlický et al. 2002). They consist of pulses or fast-drift bursts in a limited frequency range, and they slowly drift towards the lower frequencies. Therefore some of them are similar to the precursors mentioned above. Most of the DPSs have been observed during plasmoid ejections which, according to Ohyama \& Shibata (1998), can be characterized as bright soft X-ray features ejected upwards during flares. Kliem et al. (2000) proposed that each individual burst in the DPS is generated by superthermal electrons, accelerated at the peak of the electric field in the bursting regime of the magnetic field reconnection. The global slow negative frequency drift of the DPS envelope was explained by a plasmoid propagating upwards in the corona and towards the lower plasma densities. The magnetic field structure below the ejected plasmoid limits the frequency range of the DPS.

In this paper we present a multi-wavelength analysis of the features and events connected with a flare-associated halo-type CME. The CME was first observed at 11:50 UT on December 18, 2000 in the SOHO LASCO C2 coronagraph image with a speed estimate of $650 \mathrm{~km} \mathrm{~s}^{-1}$ (LASCO CME Catalog, Catholic University of America). The event was accompanied by a radio type II burst with precursor features, a type IV continuum, a partly disappearing filament, an EIT wave, and moving soft X-ray fronts. In Sect. 2 we describe the instruments, while in Sect. 3 we give a summary of the soft X-ray, EUV, $\mathrm{H} \alpha$, and white light observations. In Sect. 4 we summarize the radio and hard X-ray observations, and in Sect. 5 we discuss the results.

\section{Instruments}

In this study we concentrate on analysing $\mathrm{H} \alpha$ images from Kanzelhöhe Sonnenobservatorium (Messerotti et al. 1999), soft X-ray images from the Yohkoh (Ogawara et al. 1991) Soft X-ray Telescope (SXT; Tsuneta et al. 1991), hard X-ray images from the Yohkoh Hard X-ray Telescope (HXT; Kosugi et al. 1991), EUV images from SOHO EIT (Delaboudinière et al. 1995), white light images from $\mathrm{SOHO}$ LASCO (Brueckner et al. 1995), and radio images at decimetric/metric wavelengths from the Nançay Radioheliograph (Kerdraon \& Delouis 1997). We also use dynamic radio spectra from Ondřejov at $800-2000 \mathrm{MHz}$ and from Potsdam-Tremsdorf (AIP) at 40-800 MHz (Mann et al. 1992).

The Nançay Radioheliograph operates at five frequencies: $164,236,327,410$, and $432 \mathrm{MHz}$, and full disk radio images can be obtained with $0.1 \mathrm{~s}$ time resolution. The average cadence of the Kanzelhöhe $\mathrm{H} \alpha$ images is about one minute. SOHO EIT took Fe XII $195 \AA$ images every 12 min during the flare. Yohkoh SXT image cadence depends on the observing mode used, and in this case it varies between 1 and $10 \mathrm{~s}$. During the flare SXT was in partial frame mode. Yohkoh HXT images have spatial resolution of 5 arcsec. HXT went into flare mode with high sampling rate at 11:09 UT. Time resolution for both the Potsdam-Tremsdorf and Ondřejov spectrum is $0.1 \mathrm{~s}$.

\section{Flare- and CME-associated features in soft $\mathrm{X}$-rays, EUV, $\mathrm{H} \alpha$, and white light}

On December 18, 2000 a GOES C7.0-class X-ray flare started at 11:02 UT in NOAA Active Region 9269 (N14 E03) and peaked at 11:11 UT. NGDC/NOAA lists an $\mathrm{H} \alpha$ flare starting at 11:02 UT, with maximum intensity at 11:14 UT, and ending at 11:52 UT.

In the Yohkoh SXT soft X-ray images we see rising flare loops already at 10:57 UT, see Fig. 1. From the three available full-disk SXT images before 11:02 UT we can determine the rise speed to be about $90 \mathrm{~km} \mathrm{~s}^{-1}$. The GOES flux rise was not observed until 11:02 UT, so the flare start consisted mainly of rising loops. The small-scale brightening was not detected by GOES.

In $\mathrm{H} \alpha$ brightening of the flare site occurred in between the available images at 11:01:53 and 11:02:58 UT. The two ribbons started to separate around 11:04 UT. After 11:05:08 UT bright structures were observed to spread northwards from the flare region. At 11:06:13 UT (see Fig. 2) an $\mathrm{H} \alpha$ brightening was observed around $x=-50, y=400$ arcsec from the disk center, located very near one end of a dark $\mathrm{H} \alpha$ filament. In the next $\mathrm{H} \alpha$ image at 11:08:23 UT this part of the filament had faded and partly disappeared. The fading part is indicated by arrows in Fig. 2.

At 11:08:23 UT a small $\mathrm{H} \alpha$ brightening was observed also at location $x=60, y=500$ arcsec from the disk center. This was the largest distance where $\mathrm{H} \alpha$ brightenings were observed 

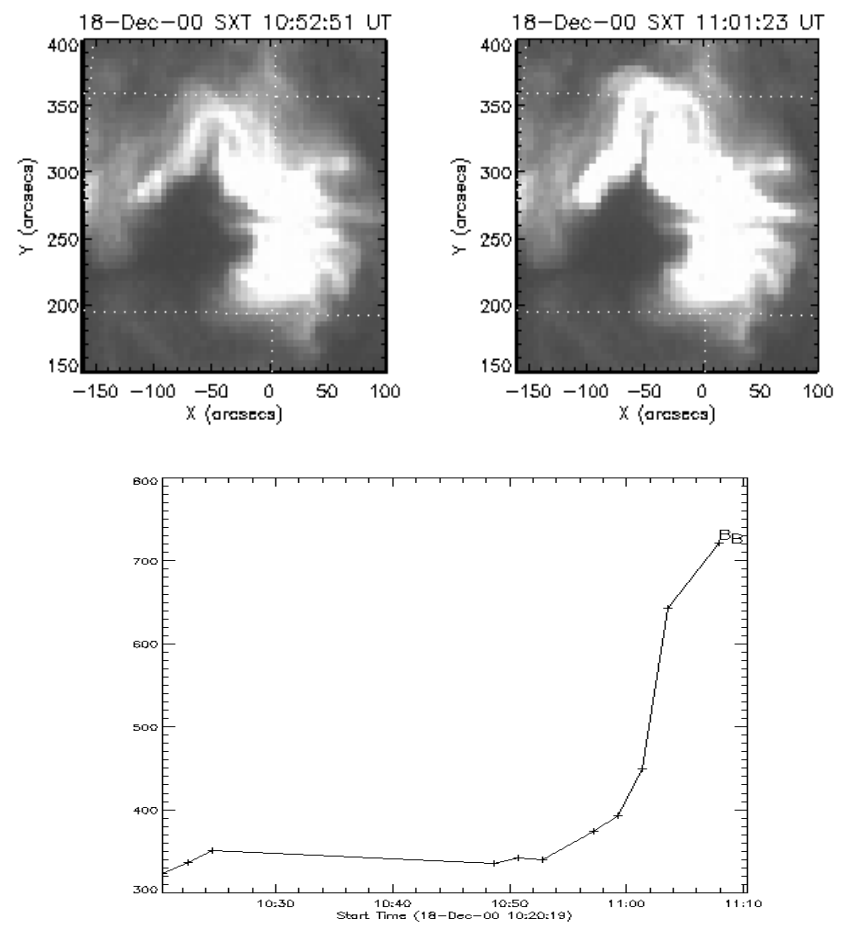

Fig. 1. Yohkoh soft X-ray images showing the rising flare loops near $x=-60, y=350$ arcsec from the disk center, at 10:52:51 UT and 11:01:23 UT. The SXT AlMg light curve of the flaring region (bottom image, in arbitrary units) shows increased brightening starting around 10:57 UT. The speed of the rising loops is about $90 \mathrm{~km} \mathrm{~s}^{-1}$ upwards. The GOES flux rise was not observed until 11:02 UT.
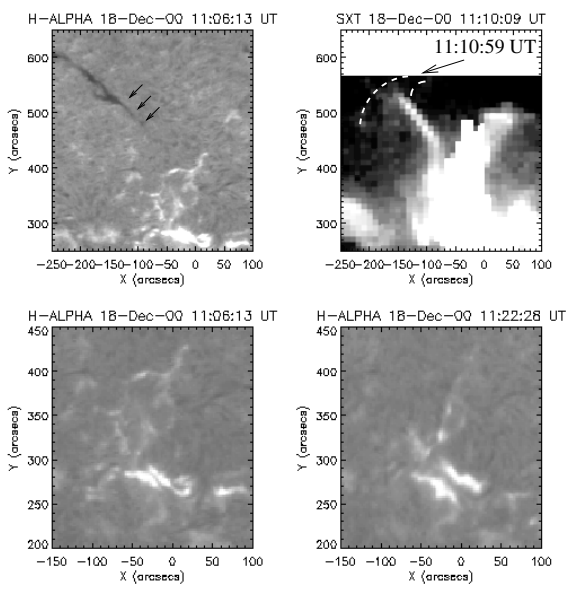
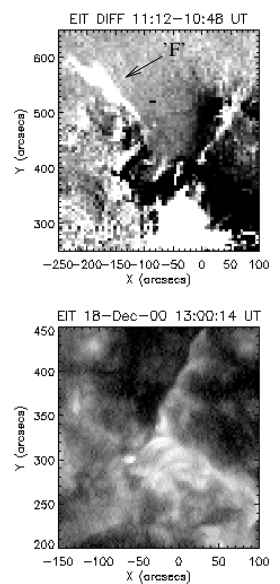

Fig. 2. Upper row: $\mathrm{H} \alpha$, soft $\mathrm{X}$-ray, and EUV images of the active region and its surroundings, field of view $x=[-250,100], y=[250$, 650] arcsec from disk center. Kanzelhöhe $\mathrm{H} \alpha$ image reveals a slowly fading and partly disappearing filament, here shown just before fading at 11:06:13 UT, fading part is indicated by arrows. The $\mathrm{H} \alpha$ brightenings were observed at 11:06:13 UT near $x=-50, y=400$, and at 11:08:23 UT near $x=60, y=500$. The dashed white lines over the Yohkoh SXT image at 11:10:09 UT show the positions of the moving soft X-ray front (front and back edges) travelling over the filament at 11:10:59 UT. EIT difference image at 11:12-10:48 UT shows the brightened, heated filament, marked with an "F". Bottom row: detailed images of the active region in $\mathrm{H} \alpha$ and EUV with the field of view $x=[-150,100], y=[200,450] . \mathrm{H} \alpha$ images show how the ribbons separate (starting around 11:04 UT). EIT image shows the post-flare loops at 13:00 UT.
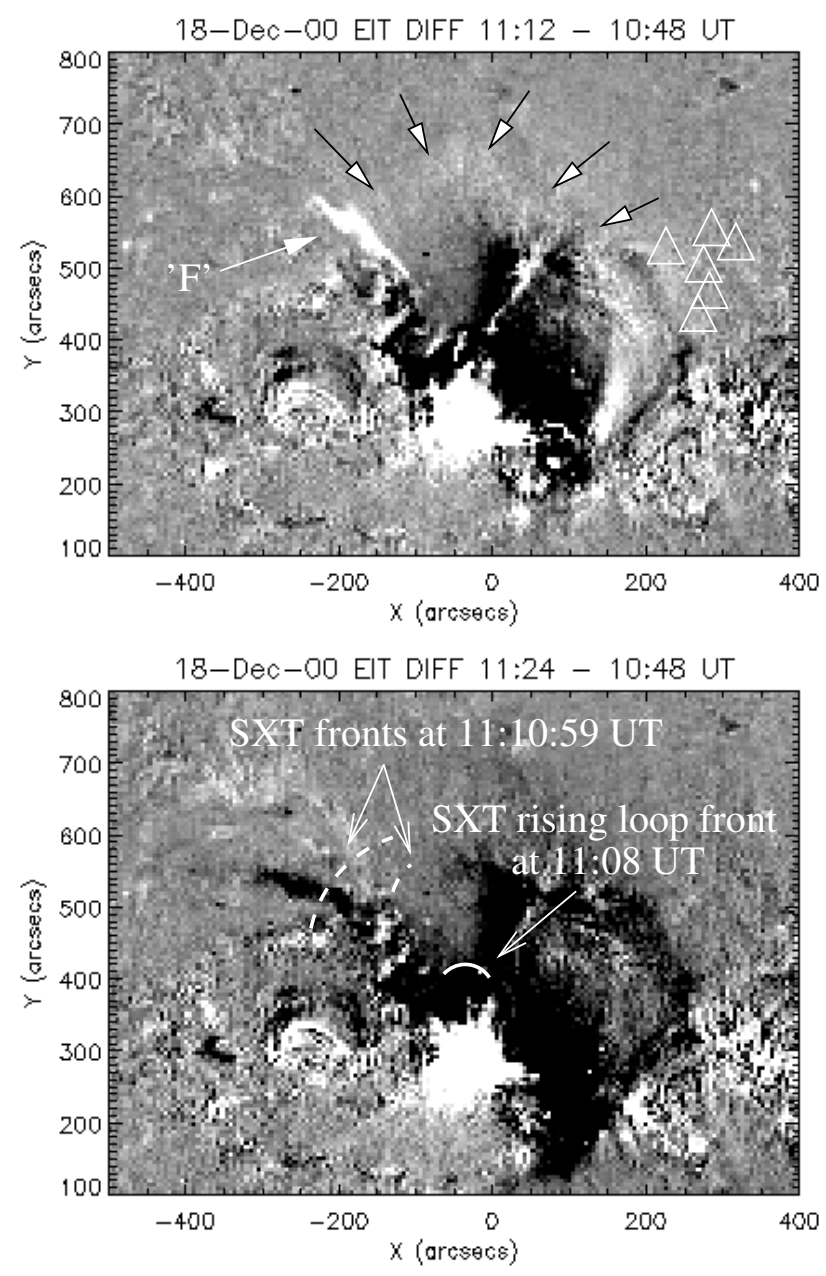

Fig. 3. At the top: EIT difference image from 11:12-10:48 UT showing the positions of the faint "EIT wave" front, indicated by the black, white-tipped arrows. The brightened filament is indicated by "F". The locations of the type II radio burst emission during 11:11:04-11:12:00 UT at $164 \mathrm{MHz}$ are shown for comparison, marked with " $\triangle$ ". At the bottom: EIT difference image from 11:24-10:48 UT showing the large dimmed regions around the active region. The dashed white lines over the image show the positions of the moving soft X-ray front at 11:10:59 UT. And the white solid line shows the extrapolated position of the soft X-ray rising loops around 11:08 UT, seen in Fig. 1.

during this flare. Figure 2 also shows the flare region and the surrounding area in soft X-rays and in EUV. Both wavelengths show how the filament location had brightened considerably between 11:05:41 UT and 11:12 UT. Yohkoh SXT full disk image at 11:07:53 UT shows both the filament channel and the area around $x=60, y=500$ very bright.

The first Yohkoh SXT flare mode images show a structure moving towards the Northeast, over the filament location, during 11:11-11:12 UT. Most probably it is an ejected loop, as we can see both the front and back end of it. The front and back edge locations of this moving soft X-ray structure at 11:10:59 UT are shown in Figs. 2 and 3 (dashed white lines). The projected speed of the front was estimated to be about $625 \mathrm{~km} \mathrm{~s}^{-1}$ (see movie in the electronic version of the Journal). 


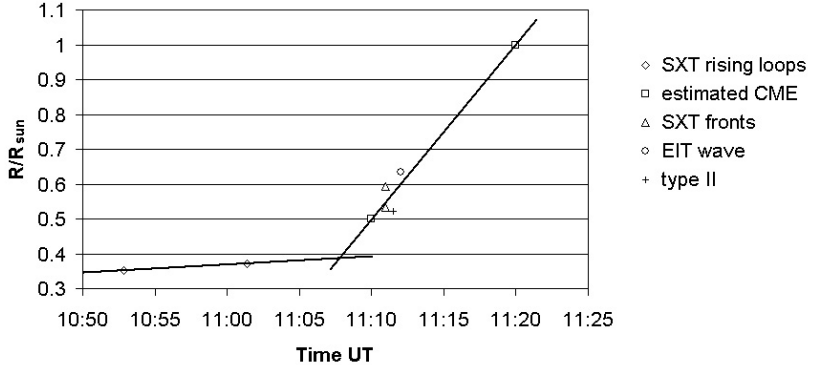

Fig. 4. The positions of the rising SXT loops $(\diamond)$, the extrapolated CME front locations $(\square)$, the SXT fronts $(\triangle)$, and the EIT wave (o) along a single radial (335 degrees). The type II position (+) is also presented but along a different radial (50 degrees). The two fitted lines intersect around 11:08 UT.
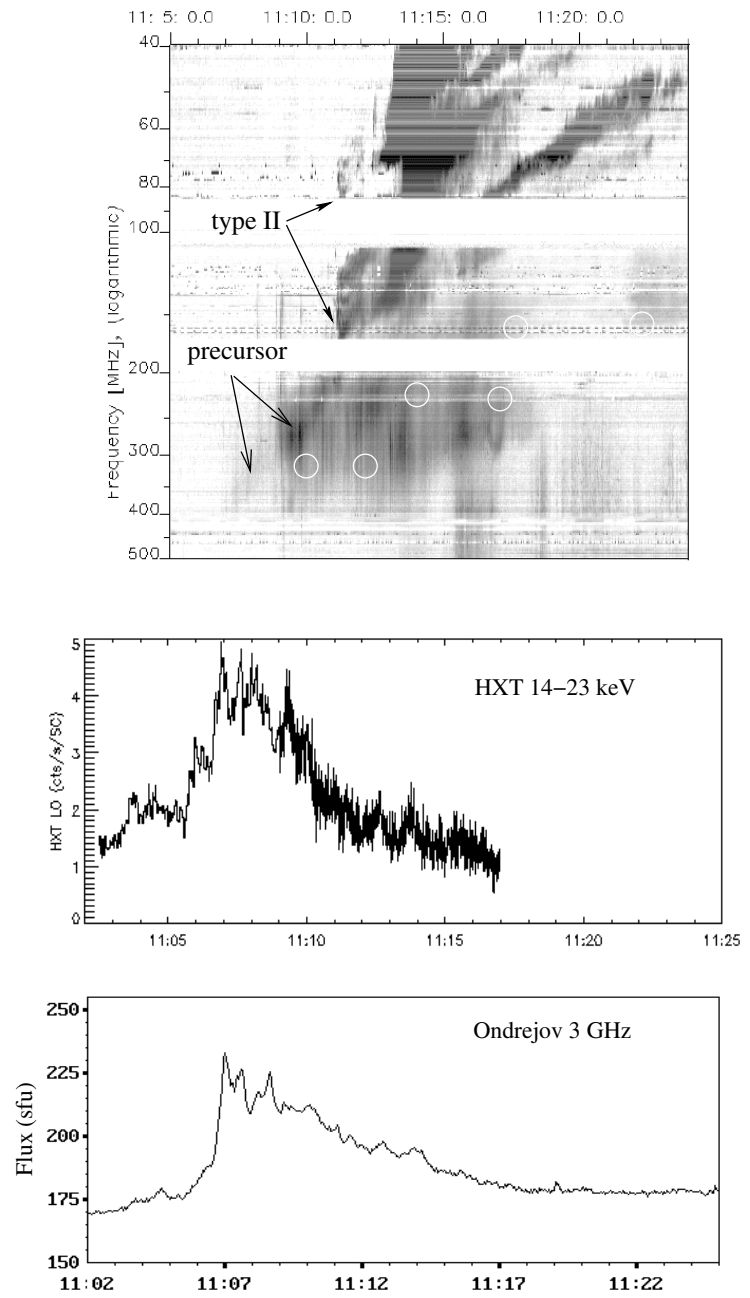

Fig. 5. AIP-Tremsdorf dynamic spectrum at $40-500 \mathrm{MHz}$, Yohkoh HXT LO channel (14-23 keV) hard X-ray counts, and Ondřejov $3 \mathrm{GHz}$ flux density observations in between 11:02 and 11:25 UT. The radio spectrum shows several type II burst lanes starting at 11:11 UT, preceded by enhanced emission and type III bursts in the $200-500 \mathrm{MHz}$ frequency range. A frequency-drifting type IV radio source is observed during 11:10-11:25 UT. Some of the type IV emission source locations are shown in Fig. 10, and the times and frequencies are indicated here with white circles.
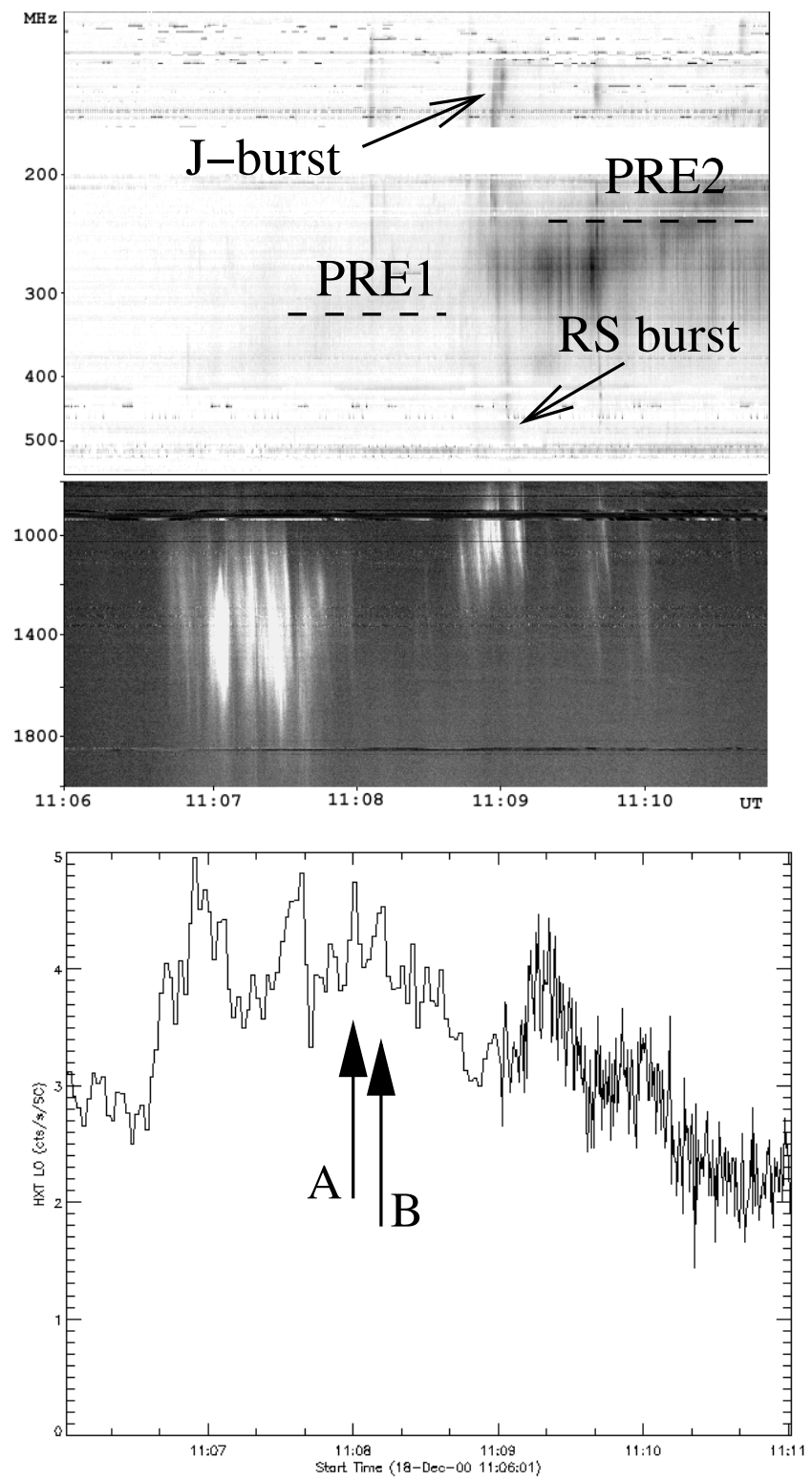

Fig. 6. Composite Potsdam-Ondřejov dynamic spectrum (40 MHz-2 GHz) and Yohkoh HXT LO channel flux curve. The spectrum shows at least one clear J-burst and several reverse drift (RS) bursts. Two precursor envelope structures PRE1 and PRE2 are also visible (imaged at 236 and $327 \mathrm{MHz}$, see Fig. 9). The decimetric spectrum at $800 \mathrm{MHz}-2 \mathrm{GHz}$ shows groups of reverse drift bursts at 1.0-1.8 GHz. The HXT LO counts shown at the bottom were mainly coming from the flare ribbons. Two distinct sources " $\mathrm{A}$ " and "B", relatively far away from the ribbons, were observed around 11:08 UT. The times are indicated here by black arrows, and the source locations are shown in Fig. 9.

An EIT wave can be seen in the EIT difference image at 11:12-10:48 UT, Fig. 3. The faint wave front is visible only in this one difference image and is indicated by arrows. The speed of the EIT wave cannot be measured accurately, as we do not know the start time. If we assume that the wave cannot have started before 11:02 UT (the time of the GOES flare start), we get a lower speed estimate of $330 \mathrm{~km} \mathrm{~s}^{-1}$. The next 


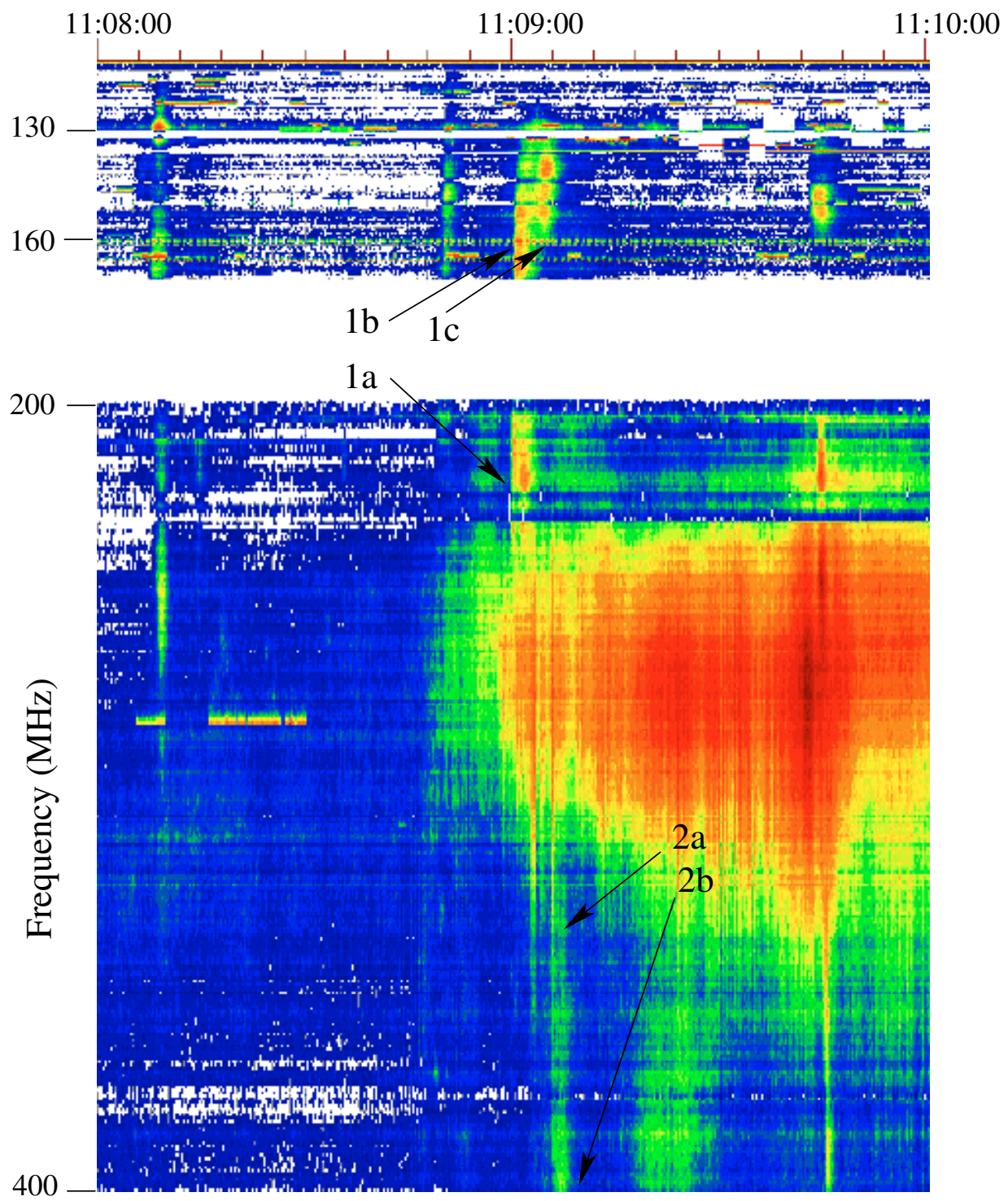

Fig. 7. One J-burst and the following reverse drift (RS) burst around 11:09 UT indicated in spectrum 1a-1c (J-burst) and 2a-2b (reverse drift burst) at Nançay imaging frequencies. The corresponding radio images are shown in Fig. 9. The radio spectrum shows how the J-burst originates at the 200 $400 \mathrm{MHz}$ frequency range (precursor envelope), turns over around $100 \mathrm{MHz}$, and then drifts towards the higher frequencies.

EIT difference image at 11:24-10:48 UT shows large dimmed regions (Fig. 3, bottom image).

In EUV, post-flare loops forming an arcade over the two ribbons started to form around 11:48 UT and are clearly visible in the 13:00 UT EIT image. The GOES flux curve goes down to pre-flare level around 12:30 UT.

A halo-type CME was first observed at 11:50 UT by the $S O H O$ LASCO $\mathrm{C} 2$ coronagraph. The estimated speed from the first observations was about $650 \mathrm{~km} \mathrm{~s}^{-1}$ (LASCO CME Catalog, Catholic University of America). From a fit to the measured CME speeds we can deduce that the CME decelerated and that the initial speed was around $700 \mathrm{~km} \mathrm{~s}^{-1}$. However, this is the CME speed in the plane of the image, and the true CME speed could be larger.

From a second order fit to the CME leading edge positions it is possible to determine the time when the CME front reached the solar limb - around 11:20 UT. We can also make a rough estimate of the time when the CME was located over the active region - around 11:08 UT. The CME front would have reached the EIT wave location (observed at 11:12 UT) around 11:12-11:14 UT. Figure 4 presents the positions of the
SXT front, the rising SXT loops, the EIT wave, and the extrapolated CME front along one radial (335 degrees, clockwise from North). The radio type II burst position is also shown in this figure, but it was visible and calculated along a different radial (50 degrees). The plot basically shows a transition from initial (slow) expansion to fast eruption. The fitted lines of these two different types of evolution intersect around 11:08 UT.

\section{Radio and hard X-ray observations}

The dynamic radio spectrum at $40-500 \mathrm{MHz}$ shows several type II lanes at the fundamental and second harmonic, as well as band-splitting and herringbone structures, see Fig. 5. The first type II burst starts at 11:11:04 UT with fundamental emission at $80 \mathrm{MHz}$ and second harmonic at $160 \mathrm{MHz}$. This burst can be followed in the spectrum only until 11:12 UT, after which several type II bursts appear in the spectrum and the lanes get mixed. The frequency drift of the first type II burst is about $0.25 \mathrm{MHz} \mathrm{s}^{-1}$ in accordance with the usual drift rate at $0.1-1.0 \mathrm{MHz} \mathrm{s}^{-1}$ (see, e.g., Cliver et al. 1999). A type IV frequency-drifting structure is also visible in the spectrum, 

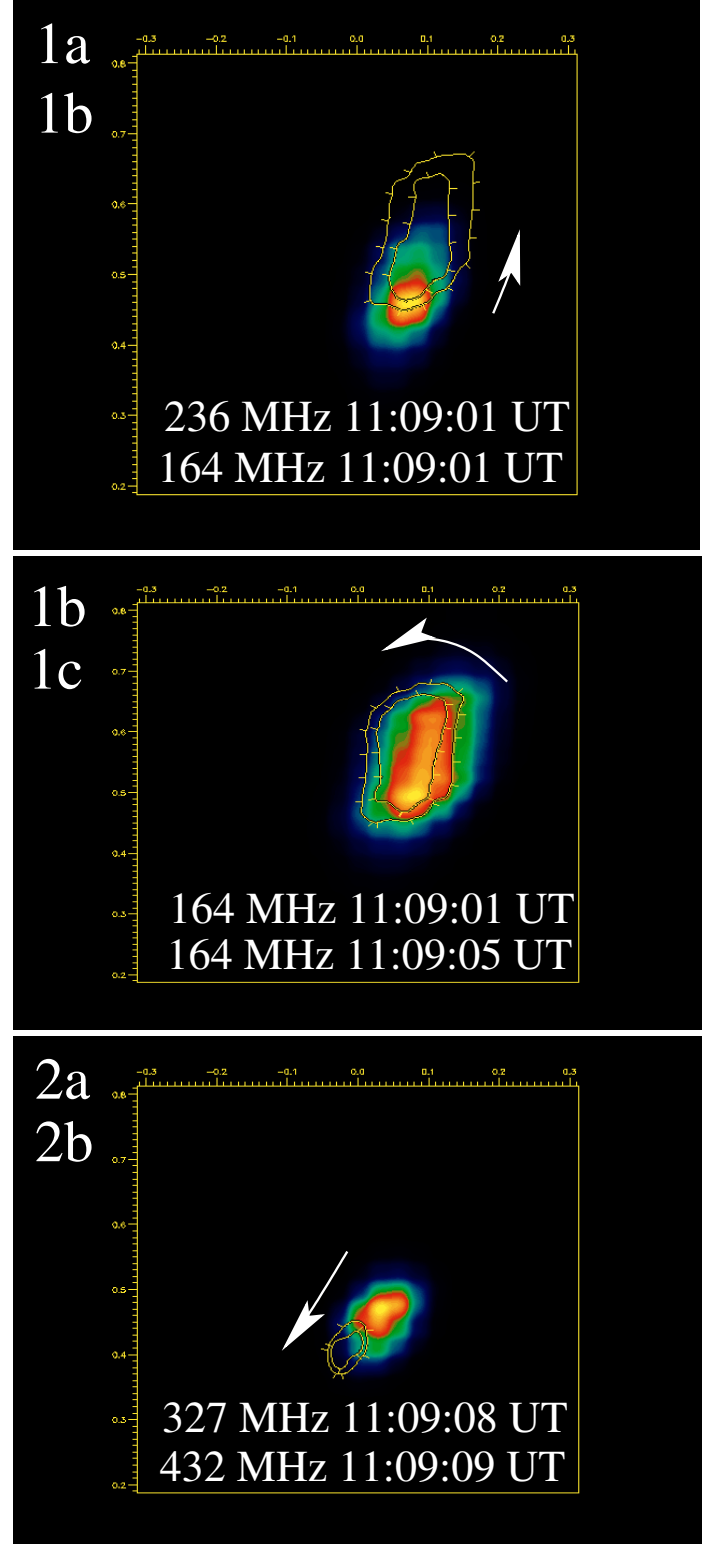

Fig. 8. Snapshots of the radio sources at selected frequencies along the J-burst $(1 \mathrm{a}-1 \mathrm{c})$ and the reverse drift burst $(2 \mathrm{a}-2 \mathrm{~b})$. Due to the large emitting source sizes, we plotted only the source centers in Fig. 9.

starting at $400 \mathrm{MHz}$ at about 11:10 UT. The emission envelope drifts slowly towards the lower frequencies. The type IV emission ends around 11:25 UT at about $100 \mathrm{MHz}$.

Before the first type II lane an "arc"-shaped precursor structure (PRE1) is seen at 400-250 MHz, starting around 11:06:30 UT (Fig. 5, shown in detail in Fig. 6). This "arc" is followed by a frequency-drifting envelope of narrowband bursts (PRE2) at 11:09-11:11 UT. The envelope drifts from $300 \mathrm{MHz}$ to about $200 \mathrm{MHz}$, ending at about the same time when the first type II burst starts. The frequency drift of the PRE1 precursor is relatively high, about $2 \mathrm{MHz} \mathrm{s}^{-1}$.

Superposed on the "arc" and on the narrow-band burst envelope are several type III bursts. Some of these bursts are J-shaped (incomplete U-bursts) with turn-over frequencies around $120 \mathrm{MHz}$, and some are reverse drift bursts (drifts towards the higher frequencies, i.e. towards the higher densities). Type III radio bursts are basically plasma emission at the local plasma frequency, and they mark the paths of accelerated electron beams. The U- and J-bursts are therefore mostly associated with closed loops (Aurass \& Klein 1997).

One J-burst trajectory is shown in Fig. 9 where the numbers $1 \mathrm{a}-1 \mathrm{c}$ indicate the observing times and frequencies marked in Fig. 6. The arrows point out the electron beam path. Immediately after the J-burst we see a reverse drift burst starting around $300 \mathrm{MHz}$ marked with the numbers $2 \mathrm{a}$ and $2 \mathrm{~b}$ over the dynamic spectrum in Fig. 6, and the burst locations at these frequencies are shown in Fig. 9.

Also at decimetric wavelengths in the frequency range 1-2 GHz, several reverse drift bursts with positive frequence drifts of about $200 \mathrm{MHzs}^{-1}$ are visible. Ondřejov dynamic spectrum (middle part of Fig. 6) shows the following burst groups: 11:06:43-11:07:50 UT at 1-1.8 GHz, 11:08:50-11:09:15 UT at 0.8-1.3 GHz, and 11:09:35-11:10:10 UT at 0.8-1.5 GHz.

Compared with hard X-rays, the decimetric radio emission starts simultaneously. Most of the hard X-ray counts originate from the flare ribbons, and the overall count rates are very low. In the bottom part of Fig. 6 we have marked two time periods, "A" and "B", when the hard X-ray sources were observed outside the flare ribbons. The dynamic spectrum shows a reversedrift type III burst at the same time at 100-320 MHz. The hard $\mathrm{X}$-ray source locations $\mathrm{A}$ and $\mathrm{B}$, imaged with $10 \mathrm{~s}$ integration time, are plotted in Fig. 9 in white contours. EIT and SXT images show that these hard X-ray sources were located at the ends of loop structures. Some of the soft X-ray loops are outlined in the image with white dashed lines. For example the projected loop length from the radio precursor site PRE1 to the hard X-ray source location B is approximately $175000 \mathrm{~km}$. Hard X-rays are mostly produced by bremsstrahlung of nonthermal electrons hitting the chromosphere or lower corona (Saint-Hilaire \& Benz 2003).

Figure 9 summarizes the locations of the radio precursors PRE1 at $327 \mathrm{MHz}$ (indicated by the circle) and PRE2 at $236 \mathrm{MHz}$ (indicated by the square), along with the locations of the radio type II burst (indicated by the triangles). The precursor sources do not move much, but due to projection effects this can simply mean that they are moving upwards (i.e., towards the observer). It should be noted that all these radio sources were located some distance away from the flare core region; the flare ribbons are visible in hard X-rays, plotted in white contours near $x=0, y=270$ in Fig. 9.

The locations of the radio type IV burst are shown over an EIT difference image in Fig. 10. The selected times and frequencies were marked in the dynamic spectrum in Fig. 5 (white circles). As the emission frequency drifts towards the lower frequencies, the source locations approach the solar limb. This is in the same direction as the main part of the CME front is moving. This is in accordance with the idea that moving type IV bursts are emitted by trapped suprathermal electrons, through either the gyrosynchrotron process or plasma emission, and that the radio sources are characterised by systematic outward motions, often in association with prominence eruptions (see, e.g. Klein \& Mouradian 2002). 

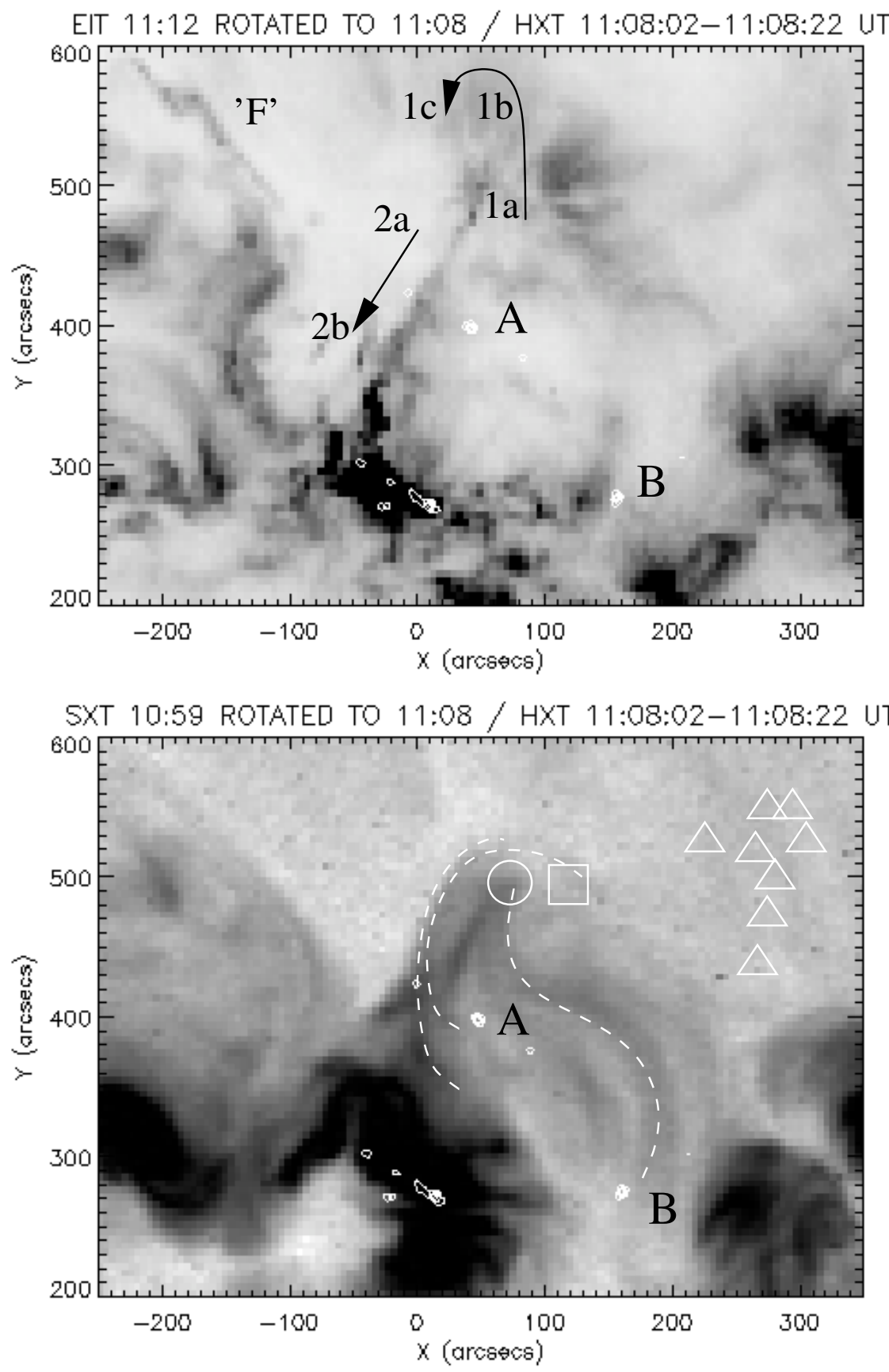

Fig. 9. Yohkoh 14-23 keV hard X-ray sources A and B (observed with $10 \mathrm{~s}$ intergration time, at 11:08:02-11:08:12 UT and 11:08:12-11:08:22 UT) plotted over the EIT (top) and SXT (bottom) images in white contours. During the flare most of the hard $\mathrm{X}$-rays were coming from the flare ribbons site (white contours near $x=0, y=$ 270 arcsec from the disk center). Locations of the emission source centers at selected times of the J-burst (1a-1c) and the following reverse drift burst $(2 \mathrm{a}$ and $2 \mathrm{~b})$ are also plotted over the EIT image at the top. The black arrows show the direction of the positional movement in time. The brightened filament in the EIT image is marked with "F". At the bottom, radio sources are plotted over the rotated Yohkoh SXT image from 10:59 UT. "o" indicates the location of the first precursor structure PRE1 at $327 \mathrm{MHz}$ during 11:07:30-11:08:30 UT, " $\square$ " indicates the location of the second precursor structure PRE2 at $236 \mathrm{MHz}$ during 11:09:15-11:11:00 UT, and " $\triangle$ "s indicate the locations of the type II burst at $164 \mathrm{MHz}$ during 11:11:04-11:12:00 UT. White dashed lines outline some of the most prominent soft X-ray loops.

\section{Discussion}

We have analysed multi-wavelength data from the December 18, 2000 flare-CME-event, in order to examine the conditions and early signatures of a CME lift-off. The halo-type CME had an initial speed of $\sim 700 \mathrm{~km} \mathrm{~s}^{-1}$, making it an intermediate between a fast and a slow CME. It was also associated with separating footpoints during a two-ribbon brightening, which in the Zhang \& Golub (2003) classification is the signature of a fast CME.

Gradual expansion was observed in the active region in soft X-rays already some $5 \mathrm{~min}$ before the flare start. The expansion was mainly due to rising loop structures with an estimated speed of $\sim 90 \mathrm{~km} \mathrm{~s}^{-1}$, similar to the expansion speed reported by Foley et al. (2003). The GOES C7.0 class flare started at 11:02 UT with simultaneous brightening in $\mathrm{H} \alpha$.

At 11:03 UT emission started in hard X-rays (14-23 keV) and at $3 \mathrm{GHz}$, indicating the presence of non-thermal particles. At 11:06:30 UT an impulsive jump in emission was observed in hard X-rays and at $3 \mathrm{GHz}$, and the first group of decimetric reverse drift bursts and the metric radio precursor simultaneously appeared in the spectrum. The simultaneous start is significant, since the hard X-ray sources were located at the flare ribbons site and the radio precursor was observed over a loop system some 230 arcsec $(160000 \mathrm{~km})$ away from the flare ribbons. Plasma emission at $327 \mathrm{MHz}$ usually requires source heights around $70000 \mathrm{~km}$, so simple projection effects cannot explain the large distance. An $\mathrm{H} \alpha$ brightening was 

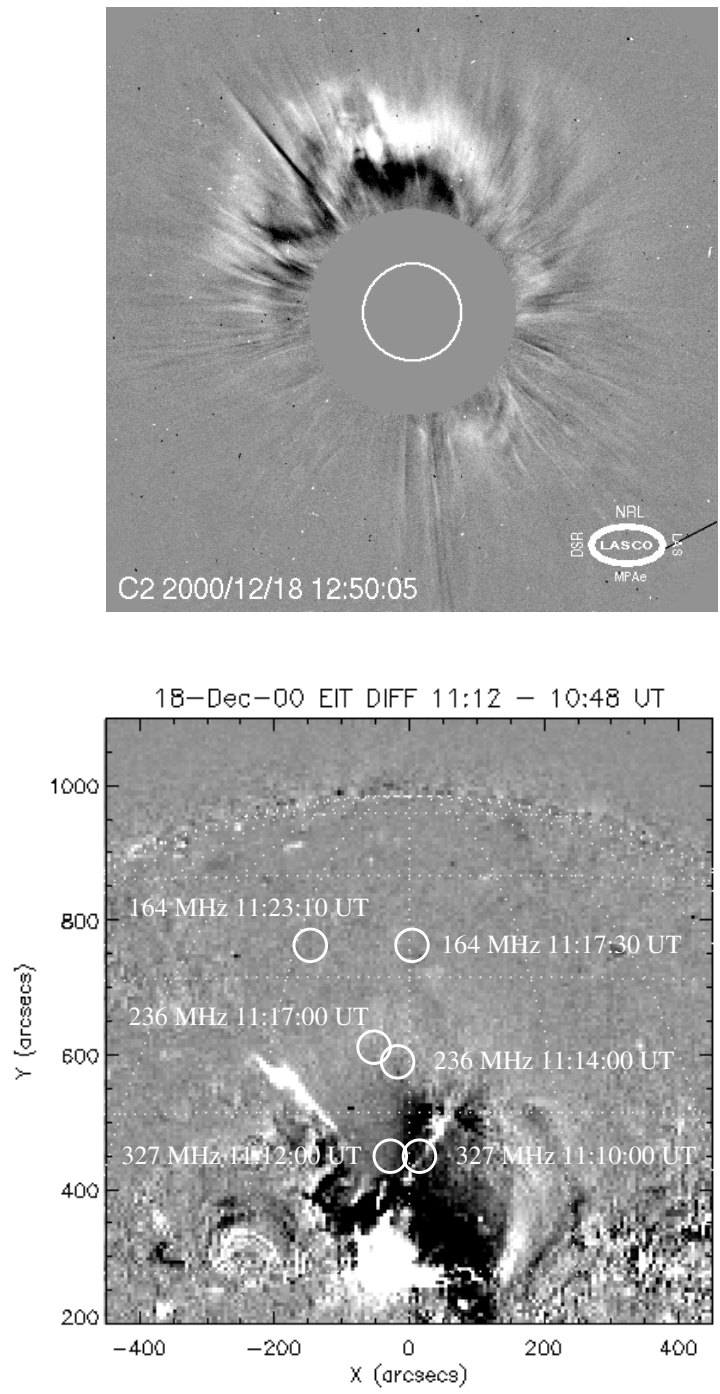

Fig. 10. SOHO LASCO $\mathrm{C} 2$ running difference image at 12:50-12:26 UT showing the halo CME on December 18, 2000. The initial speed from the 2nd order fit was estimated to be around $700 \mathrm{~km} \mathrm{~s}^{-1}$, and the average speed from the linear fit around $510 \mathrm{~km} \mathrm{~s}^{-1}$ (LASCO CME Catalog, Catholic University of America). Selected radio source locations of the type IV frequency-drifting emission (white circles) are plotted over the EIT 11:12-10:48 UT difference image at the bottom. The times were indicated by white circles in the dynamic spectrum in Fig. 5.

observed near the radio precursor location approximately at the same time and appeared between the available $\mathrm{H} \alpha$ images at 11:06:13 and 11:08:23 UT, which confirms that the flare was spatially extended near the time of the radio precursor, at least in the chromospheric level.

The nearby filament started also to fade between 11:06:13 and 11:08:23 UT. No part of this filament was located inside the flaring region, but the narrow filament end closest to the AR was very near the $\mathrm{H} \alpha$ brightenings observed at 11:06:13 UT. Both the filament channel and the area around $x=60, y=500$ were bright in soft X-rays at 11:07:53 UT. It is therefore possible that the flare initiated the heating and rise of the filament, although the moving soft X-ray front (speed estimate $625 \mathrm{~km} \mathrm{~s}^{-1}$ ) passed over the filament at approximately the time of the filament fading, and the faint EIT wave was also present at the same location at the same time.

The time-distance positions presented in Fig. 4 showed a transition from the initial slow expansion to faster ejection around 11:08 UT, which is in agreement with the timing of the $\mathrm{H} \alpha$ flare brightenings, the appearance of the radio precursor, and the heating of the filament. However, the large distances between the filament heating site and the location of the radio precursor at the same time suggest that more than one loop system was involved in the eruption. The frequency-drifting precursor envelope near $x=60, y=500$ arcsec from disc center indicates a rising structure already high in the corona. Moreover, from the J- and RS bursts we know that electron acceleration took place within this rising structure. These particle beams could be imaged and they traced large soft X-ray structures from the precursor location towards the flare core region. At certain intervals hard X-ray emission could be seen at the footpoints of some of these loops. Futhermore, the trajectories of the J-bursts and the RS bursts indicate a closed magnetic field structure. Such a configuration is expected, for example, in the plasmoid ejection model by Karlický (2004) and in the rising flux rope model presented by e.g. Forbes (1986) and Martens \& Kuin (1989).

It is commonly believed that decimetric RS bursts are generated by electron beams similar to the type III bursts. But their frequency drifts are sometimes relatively small (as in our case), and a problem arises as to how to reconcile the density height scales in the low corona with high-speed electron beams. Beam propagation along helical magnetic field structure was proposed by Karlický \& Šimberová (2002) to overcome this problem. In our case, the RS bursts were observed simultaneously with the hard X-ray sources registered far away from the flare site, and the large distances naturally explain the observed frequency drifts. Reverse drift bursts that are closely associated with a radio precursor suggest that the precursor emission comes from the site where electron acceleration takes place. As the precursor envelope drifts to lower frequencies, i.e. lower electron densities, the acceleration site is rising. Acceleration itself can be created by any of the following: a reconnection outflow termination shock (Forbes 1986; Aurass et al. 2002; Aurass \& Mann 2004), a growing shock (Vršnak \& Lulić 2000a,b), a rising X-point (Chen et al. 2004), or expanding loops (Klassen et al. 2003).

In our case no expanding loops were seen near the precursor location, although there was some indication of heating (soft X-ray loops were observed to brighten). The flare ribbons and the associated rising filament were located too far away from the radio precursor. The only evidence of a shock in EUV and soft X-rays is the EIT wave. Because of the low image cadence the true EIT wave velocity is unknown. No Moreton wave was observed in the $\mathrm{H} \alpha$ images, but often no clear $\mathrm{H} \alpha$ wave fronts are seen even if EIT waves exist (Okamoto et al. 2004).

An indication of a moving shock front is the type II radio burst near the EIT wave front. The EIT wave front is not completely spherical, and in the direction of the type II burst it 
Table 1. Summary of observed events related to the December 18, 2000 "halo" CME.

\begin{tabular}{lll}
\hline \hline Time (UT) & Event & Comments \\
\hline 10:57 & Rising soft X-ray loops & 90 $\mathrm{km} \mathrm{s}^{-1}$ (Yohkoh SXT) \\
11:02 & H $\alpha$ and GOES soft X-ray flare onset & Kanzelhöhe, 1 min image cadence \\
11:03 & Hard X-ray flare onset & Yohkoh HXT \\
11:07 & Hard X-ray maximum & \\
11:07 & Start of metric "arc" precursor & Location above loop ends (nearby negative polarity region) \\
11:07 & Start of decimetric RS bursts & \\
11:08 & Filament faded in H $\alpha$ & 180 000 km away from the flare ribbons \\
11:08 & Start of metric J- and RS-bursts & trace large loop structures \\
11:08 & Remote hard X-ray sources & location at ends of large loops \\
11:09 & Start of radio type IV emission & moving Northward \\
11:10 & Brightening along filament channel & First soft X-ray image in flare mode \\
11:11 & GOES flare maximum & \\
$11: 11$ & Start of type II radio burst & Northwest of AR \\
11:11 & Soft X-ray moving front observed & Northeast of AR, estimated speed 625 km s \\
& over the filament & \\
11:12 & Filament seen brightened in EUV & in only one of the EUV images between 11:00 and 11:24 UT \\
11:12 & EIT wave in difference image & spherical front 280 000 km away from the AR \\
$11: 14$ & H $\alpha$ flare maximum & \\
$11: 50$ & First appearance of CME & LASCO C2 bright front in North, estimated speed 650 km s \\
\hline
\end{tabular}

has not spread very far northwards. Also larger density structures are visible near the type II burst site, both in EUV and in soft X-rays. Metric (coronal) type II radio emission is thought to have at least three different types of excitation processes (see, e.g., Claßen \& Aurass 2002); it can be generated by flare-related blast wave shocks, (e.g., Pohjolainen et al. 2001), shocks driven by the leading edges of CMEs (e.g. Maia et al. 2000), or shocks driven by the internal parts or flanks of a CME (X-ray "blobs", Klein et al. 1999; expanding X-ray loops, Klassen et al. 2003). The shock driver has to have high enough speed, and in our case the estimated CME speed $\left(700 \mathrm{~km} \mathrm{~s}^{-1}\right.$ initially, decelerating later) would be sufficient to drive a shock. However, the only signature of moving material on the disk was the soft X-ray front that was moving at a speed of $625 \mathrm{~km} \mathrm{~s}^{-1}$ towards the Northeast; the opposite direction in relation to the type II burst source. The moving type IV burst locations (shown in Fig. 10) outline the most dense parts of the CME towards the North. The start time of the EIT wave cannot be determined due to the low image EIT image cadence, but if we assume that it was formed at the time of the transition from slow expansion to impulsive eruption (11:08 UT), we get a speed estimate of about $650 \mathrm{~km} \mathrm{~s}^{-1}$. The lower limit is $330 \mathrm{~km} \mathrm{~s}^{-1}$ if we assume that the wave started at the time of the flare start (11:02 UT). However, there are EIT wave models that can explain the intrinsic difference between EIT and Moreton wave speeds, and which put the EIT wave start time even earlier than the flare start (Chen et al. 2002).

To summarize the results, we find that

- The flare-halo CME event starts with slow expansion in the flare region, followed by simultaneous signs of wide-spread disturbances (heating and rise of a filament away from the flare core region, $\mathrm{H} \alpha$ brightenings, remote radio precursor).

- The radio precursor is formed by a rising structure that is closely associated with electron acceleration. Most of the bursts are reverse drift bursts, i.e. they are directed down in the solar atmosphere, and in this case they follow field lines from the precursor site towards the flare core region. The electron beams follow closed field lines that have large loop lengths up to $175000 \mathrm{~km}$. Hard X-ray emission is observed at the ends of these loops, and the decimetric reverse slope burst groups show temporal correlation with hard X-rays.

- A shock signature is observed at radio wavelengths (a frequency-drifting type II burst), as well as an EIT wave. The EIT wave front and the type II burst location are located close to each other, while no definite association can be made because of the low EIT image cadence. No X-ray or EUV ejecta can be observed in the direction of the type II burst or near the radio precursor.

- The frequency drift rate of the precursor is relatively high. High spectral drifts were also recorded during the May 2, 1998 halo CME event, where the radio sources were clearly associated with a flare blast wave and where the EIT wave and the $\mathrm{H} \alpha$ Moreton wave were cospatial (Pohjolainen et al. 2001).

- Two possibilities remain for the rising structure and accelerator of electron beams: 1) large-scale loops that connect the flare core region and the precursor site in the close vicinity of two separate rising filaments, and 2) a growing shock that accelerates electrons along closed field lines until the multipolar field is opened and the CME is lifted off. 
Acknowledgements. We thank the radio group at LESIA, Observatoire de Paris, France, for the use of Nançay Radioheliograph data. The LASCO CME catalog is generated and maintained by NASA and the Catholic University of America in co-operation with the Naval Research Laboratory. Yohkoh data are available at the DARTS database, maintained by ISAS/JAXA in Japan. SOHO is an international cooperation project between ESA and NASA. S.P. wishes to thank the Väisälä Foundation of the Finnish Academy of Science and Letters for a travel grant to Meudon, and M.K. would like to acknowledge the support by the Grant GAAS 3003202 .

\section{References}

Aurass, H., \& Klein, K.-L. 1997, A\&AS, 123, 279

Aurass, H., Vršnak, B., \& Mann, G. 2002, A\&A, 384, 273

Aurass, H., \& Mann, G. 2004, ApJ, 615, 526

Biesecker, D. A., Myers, D. C., Thompson, B. J., Hammer, D. M., \& Vourlidas, A. 2002, ApJ, 569, 1009

Brueckner, G. E., Howard, R. A., Koomen, M. J., et al. 1995, Sol. Phys., 162, 357

Caroubalos, C., Hillaris, A., Bouratzis, C., et al. 2004, A\&A, 413, 1125

Chen, P. F., Shibata, K., Brooks, D. H., \& Isobe, H. 2004, ApJ, 602, 61

Chen, P. F., Wu, S. T., Shibata, K., \& Fang, C. 2002, ApJ, 572, 99

Claßen, H. T., \& Aurass, H. 2002, A\&A, 384, 1098

Cliver, E. W., Webb, D. F., \& Howard, R. A. 1999, Sol. Phys., 187, 89

Delaboudinière, J.-P., Artzner, G. E., Brunaud, J., et al. 1995, Sol. Phys., 162, 291

Delannée, C., \& Aulanier, G. 1999, Sol. Phys., 190, 107

Eto, S., Isobe, H., Narukage, N., et al. 2002, PASJ, 54, 481

Foley, C. R., Harra, L. K., Matthews, S. A., Culhane, J. L., \& Kitai, R. 2003, A\&A, 399, 749

Forbes, T. G. 1986, ApJ, 305, 553

Glover, A., Harra, L. K., Matthews, S. A., \& Foley, C. A. 2003, A\&A, 400,759

Gopalswamy, N., Yashiro, S., Michalek, G., et al. 2002, ApJ, 572, 103

Gosling, J. T. 1997, Goronal Mass Ejections, Geophysical Monograph, 99, 9

Ji, H. S., Wang, H., Spirock, T. J., et al. 2002, Sol. Phys., 211, 221

Karlický, M. 2004, A\&A, 417, 325

Karlický, M., Fárník, F., \& Mészárosová, H. 2002, A\&A, 395, 677

Karlický, M., \& Šimberová, S. 2002, A\&A, 388, 1016

Kerdraon, A., \& Delouis, J. 1997, in Coronal Physics from Radio and Space Observations, ed. G. Trottet (New York: Springer), 192

Khan, J. I., \& Hudson, H. S. 2000, GeoRL, 27, 1083
Klassen, A., Aurass, H., Klein, K.-L., Hofmann, A., \& Mann, G. 1999, A\&A 343, 287

Klassen, A., Aurass, H., Mann, G., \& Thompson, B. J. 2000, A\&A, 141,357

Klassen, A., Pohjolainen, S., \& Klein, K.-L. 2003, Sol. Phys., 218, 197

Klein, K.-L., \& Mouradian, Z. 2002, A\&A, 381, 683

Klein, K.-L., Khan, J. I., Vilmer, N., Delouis, J.-M., \& Aurass, H. 1999, A\&A, 346, L53

Kliem, B., Karlický, M., \& Benz, A. O. 2000, A\&A, 360, 715

Kosugi, T., Masuda, S., Makishima, K., et al. 1991, Sol. Phys., 136, 17

Maia, D., Pick, M., Vourlidas, A., \& Howard, R. 2000, ApJ, 528, L49

Mann, G. 1995, J. Plasma Phys., 53, 1,109

Mann, G., Aurass, H., Voigt, W., \& Paschke, J. 1992, ESA-Journal, SP-348, 129

Mann, G., Klassen, A., Aurass, H., \& Classen, H. T. 2003, A\&A, 400, 329

Martens, P. C. H., \& Kuin, N. P. M. 1989, Sol. Phys., 122, 263

Messerotti, M., Otruba, W., Warmuth, A., et al. 1999, in Proc. ESA Workshop on Space Weather (ESA WPP 155; Noordwijk: Netherlands), 321

Moreton, G. F. 1964, AJ, 69, 145

Nelson, G. J., \& Melrose, D. B. 1985, in Solar Radio Physics, ed. D. J. McLean, \& N. R. Labrum (Cambridge: Cambridge Univ. Press), 333

Ogawara, Y., Takano, T., Kato, T., et al. 1991, Sol. Phys., 136, 1

Ohyama, M., \& Shibata, K. 1998, ApJ, 499, 934

Okamoto, T. J., Nakai, H., Keiyama, A., et al. 2004, ApJ, 608, 1124

Pohjolainen, S., Maia, D., Pick, M., et al. 2001, ApJ, 556, 421

Reames, D. V. 1999, Space Sci. Rev., 90, 413

Robinson, R. D. 1985, in Solar Radio Physics, ed. D. J. McLean, \& N. R. Labrum (Cambridge: Cambridge Univ. Press), 385

Saint-Hilaire, P., \& Benz, A. O. 2003, Sol. Phys., 216, 205

Sterling, A. C., \& Hudson, H. S. 1997, ApJ, 491, 55

Thompson, B. J., Gurman, J. B., Neupert, W. M., et al. 1999, ApJ, 517,151

Tsuneta, S., Acton, L., Bruner, M., et al. 1991, Sol. Phys., 136, 37

Vršnak, B., \& Lulić, S. 2000a, Sol. Phys., 196, 156

Vršnak, B., \& Lulić, S. 2000b, Sol. Phys., 196, 181

Warmuth, A., Vršnak, B., Magdalenić, J., Hansmeier, A., \& Otruba, W. 2004a, A\&A, 418, 1101

Warmuth, A., Vršnak, B., Magdalenić, J., Hansmeier, A., \& Otruba, W. 2004b, A\&A, 418, 1117

Zarro, D. M., Sterling, A. C., Thompson, B. J., Hudson, H. S., \& Nitta, N. 1999, ApJ, 520, L139

Zhang, M., \& Golub, L. 2003, ApJ, 595, 1251 